\title{
El diseño web y material didáctico accesible en la enseñanza universitaria
}

\section{Accessible Web Design and Documentation in University Learning}

\author{
Dr. Jaume Jaume Mayol \\ Escuela de Hotelería de las Illes Balears / Universitat de les Illes Balears, Palma de Mallorca, España \\ jaume.jaume@uib.es \\ Dr. Francisco Perales López \\ Universitat de les Illes Balears, Palma de Mallorca, España \\ paco.perales@uib.es \\ Dra. Francisca Negre-Bennasar \\ Universitat de les Illes Balears, Palma de Mallorca, España \\ xisca.negre@uib.es \\ Dr. Gabriel Fontanet Nadal \\ Universitat de les Illes Balears, Palma de Mallorca, España \\ gfontanet@uib.es
}

Resumen: Este artículo pretende dar una visión actualizada de la implantación de los procesos de diseño universal aplicados al diseño web. Para ello se presentan las pautas de accesibilidad actuales y su aplicación en el desarrollo de web accesibles y una valoración de su cumplimiento en un conjunto representativo de universidades públicas. Los resultados que se obtienen indican que el nivel de accesibilidad no cumple los requisitos de la legislación española provocando que los estudiantes universitarios experimentan grandes dificultades para acceder a la información de los espacios virtuales, provocando, en consecuencia, una baja presencia de estudiantes universitarios con discapacidad y, en definitiva, un nivel inferior de ocupación laboral con respecto a la población sin discapacidad.

Palabras clave: Accesibilidad web; Universidades españolas; igualdad de oportunidades; e-learning accesible; Accesibilidad a la documentación

\begin{abstract}
This document shows a current review of the universal web design appliance. In this sense, a general accessible web design criteria and its usage to create accessible webs are presented. Additionally, an analysis of the accessible web design level in Spanish universities are presented, in order to determine the level of compliance of the legal rules of accessible access to digital resources. Many disabled people has a lot of difficulties to study, caused by low accessible web design levels, and for this reason they have lower possibilities to work than non-disabled people.
\end{abstract}

Keywords: Accessible web design; Spanish universities, Equal Opportunities; Accessible e-learning; Accessibility to documentation 


\section{INTRODUCCIÓN}

Las Tecnologías de la Información y la Comunicación (TIC) forman parte de las estructuras, herramientas y dinámicas de las instituciones de educación superior, tanto a nivel organizativo como didáctico, e incluso cultural. La inevitable asociación entre tecnología y progreso, tecnología y modernidad, tecnología y calidad, ha hecho que las universidades, instituciones de referencia cultural e innovación, las hayan incorporado de manera rápida e ineludible a sus escenarios educativos. Sin embargo, bajo el binomio de tecnología y progreso, también se esconde el de tecnología y exclusión y, tras un primer discurso entusiasta sobre las bondades y ventajas que suponen para la sociedad y el progreso humano, la realidad también ha demostrado que pueden ser un elemento de segregación social. Es necesario estudiar de forma precisa cual es la importancia de la accesibilidad en el mundo académico, especialmente en el diseño web corporativo y en la producción de materiales didácticos por parte de los docentes y, de forma general, a los recursos electrónicos que se ponen a disposición de los estudiantes.

Según un informe de la Fundación ONCE, que se presenta en un apartado posterior, el volumen de población con discapacidad de edades comprendidas entre 16 y 64 años alcanza, en España, alrededor del 6\% de la población, es decir, alrededor de 1,8 millones de personas poseen algún tipo de discapacidad en España.

A efectos de mejorar el nivel de formación y de oportunidades laborales posteriores, es necesario asegurar que dichas personas, de manera independiente a su discapacidad, no se encuentren en inferioridad de condiciones al realizar sus estudios de formación, sea ésta obligatoria o no. En este sentido, si bien el estudio se centra en los estudios universitarios, se plantea como fundamental la realización de un seguimiento de la escolarización del alumnado con discapacidad y analizar los abandonos en las diferentes etapas educativas (Educación Primaria, Secundaria Obligatoria, Bachillerato y Universidad) en relación con las diferentes tipologías de apoyo educativo de las que se dispone durante toda la vida educativa y las facilidades que se les ofrece respecto al acceso y uso de la información y la documentación educativa, sobretodo porque la variable con más peso sobre el abandono universitario está directamente relacionada con el rendimiento académico temprano (Tuero, Cervero, Esteban y Bernardo, 2018).

Por tanto, es necesario un mayor grado de transición entre los institutos y las instituciones postsecundarias ya que, en las condiciones actuales, muchos estudiantes con discapacidad no adquieren las habilidades de autodefensa que necesitan para sobrevivir en la universidad. Estos autores sugieren que tanto la Administración como los profesores universitarios no son conscientes de sus obligaciones legales (Eckes y Ochoa, 2005), de manera muy especial en lo referente al acceso a la documentación y a los recursos electrónicos.

De esta manera, respecto al obligado cumplimiento de determinadas directrices sobre accesibilidad, se ha observado un cambio significativo respecto a la situación legal en España, en parte por requerimiento de la Unión Europea y en parte como resultado del despliegue normativo de la legislación de accesibilidad en España, constatando también avances en la sensibilización general respecto a la problemática de la accesibilidad electrónica. Por todo ello, la realización de estudios sobre la aplicación de estas directrices debe ocupar un puesto relevante, tanto para la Administración como para el profesorado universitario (Ribera y otros, 2009).

La accesibilidad en centros formativos se puede plantear desde un punto de vista físico o lógico. En el primer aspecto se tienen principalmente en cuenta elementos arquitectónicos

El diseño web y material didáctico accesible en la enseñanza universitaria. J. Jaume

Mayol, F.J. Perales López, F. Negre Bennasar, G. Fontanet Nadal.

Página 2 de 19 
de acceso a las instalaciones del centro. Para la consideración de la accesibilidad desde el punto de vista lógico es necesario considerar la posibilidad de los estudiantes de acceder a los contenidos digitales. En este caso la accesibilidad se puede ver desde dos perspectivas:

- Acceso a la información general de los centros académicos, que aparece en la web de las diversas organizaciones.

- Acceso a los contenidos de las asignaturas y módulos formativos, una vez el alumno está matriculado y cursando las materias de los respectivos planes de estudio.

Para los autores Casasola y otros (2017, p.2), la accesibilidad web "significa no sólo poder navegar, sino también interactuar y poder aportar contenidos", entendiendo que el objetivo de la accesibilidad es conseguir adaptar y acomodar de forma que cualquier persona pueda acceder a la información y a la documentación disponible en Internet.

Desde otro punto de vista, otros autores definen la accesibilidad web como "una métrica de la Ingeniería Web que trata de elementos relacionados con la codificación y la presentación de información en el diseño y funcionalidad de un sitio web, que va a permitir que las personas con algún tipo de discapacidad puedan percibir, entender, navegar e interactuar de forma efectiva en la web, así como crear y aportar contenido" (Sam-Anlas y Stable-Rodríguez, 2016).

Resulta relevante, por tanto, para las personas con discapacidad ya que del nivel de cumplimiento de las pautas de accesibilidad dependerán sus posibilidades de participación social y educativa.

En el presente documento se analizan los niveles de accesibilidad web en centros académicos de formación universitaria.

\section{ANTECEDENTES}

\subsection{Volumen y nivel de formación y ocupación de la población con discapacidad}

Considerando la población de edad comprendida entre 16 y 64 años, el volumen de población con discapacidad en España es de aproximadamente 1,8 millones de personas, siendo casi el 6\% de la población total. Debe destacarse, en este sentido, que los volúmenes de discapacidad aumentan con la edad, según se desprende de los resultados de un informe del Observatorio sobre Discapacidad y Mercado de Trabajo en España, de la Fundación ONCE. En el caso de la población de edades comprendidas entre 16 y 24 años el porcentaje es de un $4,8 \%$ de población con discapacidad. Dicho intervalo de edades es especialmente importante en el presente estudio, puesto que precisamente es en este rango de edades donde se encuentra la mayor cantidad de personas que se están formando en centros académicos de nivel medio (institutos, colegios, etc.) o superior. El resumen de las informaciones se puede apreciar en la Tabla 1 (ODISMET, 2016).

Tabla 1.

Volumen y porcentaje de población con discapacidad en España, por edad

\begin{tabular}{ccc} 
Intervalo de edad & Total & Porcentaje \\
\hline 16 a 24 & 84.500 & $4,8 \%$ \\
\hline 25 a 44 & 455.900 & $25,7 \%$ \\
\hline 45 a 64 & 1.234 .400 & $69,6 \%$ \\
\hline
\end{tabular}

Nota: Adaptación a partir de ODISMET, 2016

El diseño web y material didáctico accesible en la enseñanza universitaria. J. Jaume

Mayol, F.J. Perales López, F. Negre Bennasar, G. Fontanet Nadal. 
Por tipo de discapacidad, la mayoría de las discapacidades poseen un componente físico $(64,4 \%)$. Los otros motivos de discapacidad son los intelectuales $(10,2 \%)$, mentales $(15,7 \%)$ y sensoriales $(9,8 \%)$, (ODISMET, 2016).

En resumen, el perfil de una persona con discapacidad en España es el de un varón de edad comprendida 45 y 64 años, con una discapacidad física y un nivel de afectación comprendido entre el $33 \%$ y el $44 \%$, que vive en un hogar con ingresos y en un área densamente poblada (ODISMET, 2016).

En cuanto a los niveles de formación, debe destacarse que el nivel formativo de las personas con discapacidad es menor al de la población sin discapacidad. Por ejemplo, el $5,8 \%$ de la población con discapacidad no tiene estudios, frente al $0,5 \%$ de la población sin discapacidad. En cuanto a la educación superior, el porcentaje de población con discapacidad con dicho nivel formativo es de un $15,1 \%$, la mitad que en el caso de la población sin discapacidad (33,2\%). Dichos porcentajes se pueden apreciar en la Tabla 2

(ODISMET, 2016).

Tabla 2 .

Niveles formativos de las personas con y sin discapacidad

\begin{tabular}{lcc} 
Nivel formativo & Población con discapacidad & Población sin discapacidad \\
\hline Sin estudios & $5,8 \%$ & $0,5 \%$ \\
\hline Educación Primaria & $23,9 \%$ & $9,6 \%$ \\
\hline Educación Secundaria & $55,2 \%$ & $56,7 \%$ \\
\hline Educación Superior & $15,1 \%$ & $33,2 \%$ \\
\hline
\end{tabular}

Nota: Adaptación a partir del ODISMET (2016)

A nivel de inserción laboral entre la población con discapacidad existe una relación directa entre el nivel de formación y la ocupación. Así, el 87,2\% de las personas con discapacidad que trabajan posee un nivel de estudios de Educación Secundaria o Superior. En particular, el sector que prevalece es el de Educación Secundaria, como se puede ver en la Tabla 3 (ODISMET, 2016).

Tabla 3.

Niveles de ocupación de la población con discapacidad, por nivel de estudios

\begin{tabular}{lc} 
Nivel de estudios & Porcentaje de población ocupada \\
\hline Sin estudios & $0,8 \%$ \\
\hline Educación Primaria & $12 \%$ \\
\hline Educación Secundaria & $59 \%$ \\
\hline Educación Superior & $28,2 \%$ \\
\hline
\end{tabular}

Nota: Adaptación a partir del ODISMET (2016)

En cuanto a niveles de ocupación, el porcentaje de población con discapacidad que trabaja es del $23,4 \%$. Ello se traduce en que sólo una de cada cuatro personas con discapacidad tiene empleo. Además, el $60 \%$ del total del colectivo de parados de larga duración son personas con discapacidad (ODISMET, 2016)

\subsection{Importancia de la accesibilidad en el mundo académico}

La importancia de garantizar el acceso de las personas con discapacidad a los sistemas formativos de educación superior se deriva del hecho de que es un requisito para su incorporación en la sociedad del conocimiento. Para Pastor y Antón (2009), la 
accesibilidad a los servicios que se ofrecen en Internet y en general a las TIC y a la Comunicación es, no sólo una necesidad para las personas con discapacidad, también un derecho. La universidad, según Muntaner y otros (2008), debe posibilitar el acceso a la formación superior poniendo al alcance del alumnado los recursos que posibiliten y potencien la continuidad en los estudios y debe ser capaz de adaptarse a la pluralidad de situaciones que se manifiestan en nuestra sociedad. Resulta sorprendente, por tanto, que no se sigan los criterios de accesibilidad en toda la documentación y los recursos electrónicos que la Institución pone a disposición de la comunidad educativa, considerando además que la presencia de las TIC en el proceso formativo es cada vez mayor debido a "la inclusión de cursos en modalidad virtual en el proceso formativo" (Amado-Salvatierra, Hilera y Otón, 2018, p. 349).

En la universidad, además de en todo el sistema educativo en general, la atención al alumnado con discapacidad se fundamenta en los principios de normalización e integración, en la provisión de servicios y en el análisis de contextos. Por tanto, estos elementos deben ser objeto de reflexión y desarrollo. En este sentido, se pueden identificar dos frentes de actuación como respuesta de la universidad al alumnado con discapacidad: la atención al desarrollo de sus capacidades e intereses y la facilitación de los elementos de acceso y dominio de situaciones con los que alcanzar niveles de igualdad con sus compañeros. El objetivo es "la creación y favorecimiento, en suma, de acciones encaminadas a compensar la discapacidad y desarrollar la capacidad de la persona" (Luque y otros, 2005). Para Casasola y otros (2017), una de los aspectos a mejorar es el acceso a la información disponible en la web. Es importante constatar que al mejorar la accesibilidad a los servicios que ofrece internet no se produce un avance exclusivamente dirigido a los estudiantes con alguna discapacidad. La accesibilidad web debe entenderse como un avance aprovechable y beneficioso para todos los usuarios.

La solución para los estudiantes universitarios con discapacidad no debe tratarse simplemente de una respuesta individual ante una situación concreta, sino que se deben ofrecer soluciones contextuales (accesibilidad y supresión de barreras) y sociales (actitudes hacia la integración), con el objetivo de cumplir los principios de igualdad de oportunidades (Luque y otros 2005). Lo contrario puede suponer una discriminación directa al tratar a este colectivo de forma menos favorable que al resto de estudiantes y también una discriminación indirecta debida a una decisión institucional que ocasiona situaciones de desventaja a un determinado grupo de personas.

En este contexto, el acceso a las TIC supone, para muchos estudiantes con discapacidad, un recurso que puede servir de elemento integrador y democratizador del acceso a la información, aunque la imposibilidad de acceder a ellas puede convertirse en una forma de marginación y exclusión social. Es por todo ello por lo que la accesibilidad a los medios tecnológicos es imprescindible para garantizar la igualdad de oportunidades y mejorar la calidad de vida de todos los estudiantes (Antón, 2010), ofreciendo no solo soluciones contextuales y sociales (Luque y otros 2005), también soluciones institucionales dirigidas a potenciar el reconocimiento positivo de las diferencias y a integrarlas como un valor añadido de la propia institución.

Atendiendo a la importancia de proporcionar las mismas oportunidades a todos los estudiantes, independientemente de su situación personal, se realizó un estudio para valorar los efectos de la legislación española en accesibilidad y de las campañas de sensibilización desde el año 2001 al 2006 (Ribera y otros, 2009, Pastor y Antón, 2008), evidenciando que los efectos han sido menores de los esperados y considerando las mejoras en accesibilidad como decepcionantes. Aun así, se valoran positivamente los

El diseño web y material didáctico accesible en la enseñanza universitaria. J. Jaume 
logros de los últimos años, destacando precisamente el respaldo legislativo que instituye la igualdad de oportunidades dirigido a evitar la discriminación y asegurar la participación plena y efectiva en el ámbito universitario.

Resulta evidente que las universidades deben favorecer, a la comunidad universitaria con algún tipo de necesidad especial, la disponibilidad de medios, apoyos y recursos para asegurar la no discriminación. Estas medidas deben incluir, entre otros servicios, los espacios virtuales posibilitando tanto su ingreso como su permanencia (Pastor y Antón, 2009), pero también su participación y el acceso a todos los recursos electrónicos. En la sociedad del conocimiento no es posible ignorar las propuestas educativas basadas en campus virtuales y que una buena parte de la docencia se realiza en aulas virtuales, ya sea de forma presencial o totalmente a distancia y/o, como ocurre en muchos campus universitarios, combinando ambas modalidades. No es por tanto posible eludir esta realidad y postergar iniciativas que tengan como objetivo ofrecer las mismas oportunidades a todos los estudiantes. En este sentido es importante destacar que las limitaciones de acceso pueden darse no solo en la formación mediante plataformas virtuales, también en los procesos de matriculación y gestión administrativa (Toledo y otros, 2013) y, de forma general, al uso de toda la documentación electrónica. Hoy en día, prácticamente todas las universidades gestionan su información con sistemas informáticos, pero todavía hoy no se valora convenientemente la importancia de procurar el acceso y uso de esta documentación a todos los usuarios.

Los servicios virtuales se han hecho cada vez más presentes en la trayectoria educativa observándose un incremento en los centros de enseñanza superior, pasando de métodos tradicionales de carácter presencial y síncrono a espacios virtuales. La accesibilidad, por tanto, juega un papel muy importante en el diseño, no solo de las páginas web, también de los cursos y de los Entornos Virtuales de Formación (Burgstahler, 2002) y de todos los recursos electrónicos que se ponen a disposición de los estudiantes. Estas modalidades de formación pueden ofrecer importantes beneficios para los estudiantes y también para los profesores con discapacidad (Lance, 2002). Bajo nuestro punto de vista esto es así, siempre y cuando se cumplan los requisitos de Accesibilidad Web.

Cabe destacar que las limitaciones para el acceso a contenidos web pueden ser debidas a deficiencias visuales, auditivas o motrices, además de las cognitivas y de lenguaje. En el caso de los estudios universitarios los principales tipos de discapacidad son los motrices, seguidos de los auditivos y los visuales. En relación a la accesibilidad web, los principales problemas de acceso web se presentan principalmente para las personas con visuales y auditivos (Guash, 2010; López, 2010; Saldarriaga, 2014).

Así pues, la aplicación de recursos tecnológicos para trabajos de colaboración, investigación y divulgación de la información hace que los entornos de aprendizaje (presenciales, semipresenciales o a distancia) posibiliten un sistema de aprendizaje flexible, abierto y activo, con altos niveles de autonomía y libertad. La accesibilidad a estos recursos es, en consecuencia, un valioso elemento para que los estudiantes con discapacidad puedan graduarse en las universidades (Antón, 2010). Las limitaciones de acceso, en cambio, suponen poderosas barreras para que la educación del siglo XXI se ofrezca en igualdad de condiciones.

La Accesibilidad web, es, por tanto, una forma de reconocer la singularidad de cada estudiante y debería suponer un compromiso para todos los profesionales de la educación y la documentación.

El diseño web y material didáctico accesible en la enseñanza universitaria. J. Jaume 


\subsection{Accesibilidad web}

El concepto de Accesibilidad Web está vinculado al objetivo de permitir que cualquier usuario de Internet, independientemente del tipo de hardware, software o condición personal (cultural, social) o capacidad, pueda acceder a los recursos contenidos en Internet (Caldwell y otros 2008).

La organización W3C (World Wide Web Consortium ${ }^{1}$ ) creó una iniciativa, la WAI (Web Accessibility Initiative - Iniciativa de Accesibilidad Web), con el objetivo de definir unas pautas de diseño de páginas web que permitan el acceso a las personas con discapacidad. Dentro del conjunto de acciones desarrolladas en la WAI, en el año 2008 se publica la WCAG 2.0 (Web Content Accessibility Guidelines). Dicha guía sustituye la anteriormente publicada WCAG 1.0, publicada en 1999. En ambos casos se trata de un conjunto de pautas de desarrollo de sitios web accesibles. Con posterioridad a la realización del presente estudio se ha publicado, en 2018, una nueva guía de diseño web accesible denominada WCAG 2.1 (Caldwell y otros, 2008; SGAD2, 2018).

Asimismo, en la iniciativa de la W3C se definen, además de pautas de accesibilidad, otras técnicas y herramientas de evaluación y reparación de la accesibilidad Web (Caldwell y otros, 2008).

Las recomendaciones que se describen en la WCAG 2.0 están organizadas en un conjunto de criterios (Caldwell y otros, 2008):

- Un conjunto de Principios de Diseño: Se pueden considerar como las bases a que se refiere cada una de las recomendaciones de la WCAG. El W3C define cuatro principios de diseño y su significado de la siguiente forma: Perceptible (la información y los componentes de la interfaz de usuario deben ser mostrados a los usuarios en formas que ellos puedan entender), Operable (Los componentes de la interfaz de usuario y la navegación debe ser manejable), Comprensible (La información y las operaciones de usuarios deben ser comprensibles, por ejemplo, haciendo el contenido de texto legible y comprensible o mediante una asistencia a la entrada de datos) y Robusta (el contenido deber ser suficientemente robusto para que pueda ser bien interpretado por una gran variedad de agentes de usuario, incluyendo tecnologías de asistencia.).

- Unos Criterios de Éxito: Se trata de criterios a seguir para alcanzar los Principios de accesibilidad comentados anteriormente. El objetivo de la consecución de los Criterios de Éxito es alcanzar los diversos niveles de accesibilidad que se definen: A, AA y AAA (descritos más adelante en el documento).

- Técnicas de consecución de los Criterios de Éxito: Son las técnicas que se recomiendan para conseguir el éxito en la programación de HTML que supere los Criterios de Éxito.

La WCAG 2.0 vincula cada uno de los Criterios de Éxito con un nivel de accesibilidad. Así, los hay que son necesarios para alcanzar un mínimo de accesibilidad, que son los que estarían vinculados al nivel A (nivel mínimo). Los niveles de accesibilidad se describen a continuación (Caldwell y otros, 2008).

\footnotetext{
${ }^{1}$ https://www.w3.org/
}

El diseño web y material didáctico accesible en la enseñanza universitaria. J. Jaume 
- Nivel A: Se alcanza cuando se cumplen todos los Criterios de Éxito que están relacionados con el nivel A. Es el nivel mínimo de accesibilidad y no lograrlo hará que un grupo de usuarios no podrá acceder al contenido de la web.

- Nivel AA: Se alcanza cuando se cumplen todos los Criterios de Éxito que están relacionados con los niveles A y AA. Es el nivel intermedio de accesibilidad y no lograrlo hará que un grupo de usuarios tendrá muchas dificultades para acceder al contenido web.

- Nivel AAA: Se alcanza cuando se cumplen todos los Criterios de Éxito que están relacionados con los niveles A, AA y AAA. Es el nivel máximo de accesibilidad y no lograrlo hará que un grupo de usuarios tendrá algunas dificultades para acceder al contenido web. Una web que alcanza el nivel AAA puede ser accedida por todos los usuarios

Los errores en criterios de éxito de accesibilidad web se pueden clasificar en dos tipos (Serrano 2009):

- Aquellos que se pueden detectar de forma automática, mediante un programa. Existen aplicaciones informáticas y sitios web de evaluación de los niveles de accesibilidad.

- Aquellos que sólo se pueden detectar de forma manual, con la intervención de personal especialista en temas de accesibilidad.

Se puede destacar, por ejemplo, la herramienta Test de Accesibilidad Web (TAW ${ }^{2}$ ). El TAW es una herramienta de análisis automático de la Accesibilidad Web, desarrollada por la Fundación Centro Tecnológico de la Información y la Comunicación (CTIC). La herramienta, orientada principalmente a profesionales del diseño y desarrollo web, permite analizar y detectar errores en diseño de páginas web accesibles, además de generar una lista de existencia de posibles problemas de tipo manual, que la persona evaluadora debe confirmar o descartar.

Dado que los principios de diseño accesible se plantean para cualquier tipo de usuario, la utilización de las técnicas descritas en la WAI permite que todos los usuarios de la WWW obtengan beneficios adicionales cuando se diseña de acuerdo a las normas de diseño web accesible. Entre dichos beneficios se pueden destacar (Fraiz 2008, Fontanet 2011, Jaume y otros 2011, Serrano 2009):

- Un incremento de la cuota de mercado y alcance de la audiencia, dado que la población con discapacidad puede superar el $20 \%$ en algunas regiones.

- Una mejora de la usabilidad para los visitantes, tanto si padecen una discapacidad como si no. La mejora se concreta en una navegación más clara y consistente y en una independencia del dispositivo (PC, tabletas, móvil). Este beneficio se constata en funcionalidades basadas en dispositivos móviles (Jiménez, 2010).

- Constituye un soporte para niveles de baja alfabetización, dado que la observancia de las recomendaciones de la WCAG permite facilitar la comprensión del contenido del sitio web, tanto a los usuarios con bajo nivel de alfabetización como a aquellas personas poco acostumbradas al lenguaje propio de las páginas web.

\footnotetext{
${ }^{2}$ http://www.tawdis.net
}

El diseño web y material didáctico accesible en la enseñanza universitaria. J. Jaume 
- Una mejor asistencia para el al acceso de los usuarios con reducido ancho de banda

- La reducción de los costes de desarrollo y mantenimiento del website.

- Un incremento en la reutilización de contenido.

- Una demostración de Responsabilidad Social Corporativa.

\section{METODOLOGÍA}

\subsection{Objetivos}

El proyecto realizado plantea varios objetivos:

- En primer lugar, determinar los niveles de accesibilidad web existentes en los sitios web de educación universitaria. Se han considerado los errores que se pueden detectar mediante herramientas automáticas de detección de errores, que se complementa con un análisis manual en los casos en que las herramientas automáticas detecten presuntas anomalías en el cumplimiento de las normas de Accesibilidad.

- El resultado del análisis web anterior permite obtener una perspectiva general para poder calibrar, en primer lugar, el nivel de cumplimiento de las normas de diseño web. Adicionalmente, se convierte en un punto de partida para conocer los defectos de diseño y poder corregirlos. Para poder alcanzar este segundo objetivo se propone seguir las recomendaciones de una guía de diseño de materiales académicos accesibles (Chicaiza y otros, 2014; Hilera y otros, 2015; Sama y Sevillano, 2012; Térmens y otros, 2008).

\subsection{Metodología de trabajo}

Diversos autores han estudiado la accesibilidad web de universidades españolas, hecho que confirma el interés por conocer el grado de cumplimiento de la accesibilidad por las universidades. De los resultados obtenidos se desprende que existen deficiencias de aplicación de las pautas de accesibilidad en los portales web de universidades españolas (Caballero y otros, 2010; Zubillaga, 2010; Hilera y otros, 2013; Chacón y otros, 2013; Toledo y otros, 2013; Casasola y otros, 2017)

Como complemento a estos trabajos y a efectos de poder determinar los niveles de accesibilidad en sitios web educativos se han seleccionado diferentes universidades de España. La selección se ha basado en los siguientes criterios:

- Se ha elegido el ámbito universitario, puesto que la comparación se debe realizar con la Universitat de les Illes Balears, institución en la que se ha desarrollado el proyecto.

- Se han seleccionado páginas web de las 15 mejores universidades, además de la Universitat de les Illes Balears, desde el punto de vista de la docencia, que aparecen en el documento "U-ranking (Indicadores Sintéticos del Sistema Universitario Español)"3, realizado en 2017. La Tabla 4 muestra la lista de universidades consideradas en el estudio (U-Ranking 2017).

\footnotetext{
${ }^{3}$ http://www.u-ranking.es/analisis.php
}

El diseño web y material didáctico accesible en la enseñanza universitaria. J. Jaume Mayol, F.J. Perales López, F. Negre Bennasar, G. Fontanet Nadal. Página 9 de 19 
RED. Revista de Educación a Distancia. Núm. 59, Artíc. 06, 30-10-2019

DOI: http://dx.doi.org/10.6018/red/60/06

Tabla 4.

Tabla de universidades analizadas

\begin{tabular}{ll} 
Abreviatura & Universidad \\
\hline UPF & Pompeu Fabra \\
\hline UC3M & Carlos III \\
\hline UPC & Politècnica de Catalunya \\
\hline UPV & Politècnica de València \\
\hline UAM & Autónoma de Madrid \\
\hline UC & Cantabria \\
\hline UAB & Autónoma de Barcelona \\
\hline URV & Rovira i Virgili \\
\hline UNAV & Navarra \\
\hline UMH & Miguel Hernández de Elche \\
\hline UPM & Politécnica de Madrid \\
\hline USC & Santiago de Compostela \\
\hline UB & Barcelona \\
\hline UV & València \\
\hline UAH & U. Alcalá de Henares \\
\hline UIB & U. Illes Balears \\
\hline
\end{tabular}

Nota: Elaboración propia a partir de la clasificación del U-Ranking de 2017

Siguiendo las recomendaciones del Observatorio de Accesibilidad del Ministerio de Hacienda y Función Pública (MINHAP), se ha procedido a seleccionar un conjunto de páginas web de cada uno de los sitios web, a efectos de poder evaluar su accesibilidad (SGAD, 2018). Para conseguir una homogeneidad, la selección se ha realizado de manera manual (siguiendo las mismas recomendaciones del MINHAP), eligiendo páginas web con contenidos estén presentes en todos los sitios web. De esta manera se ha procedido a:

- Elegir un total de tres páginas web de cada uno de los sitios. En total se han seleccionado 48 web.

- En cuanto a la tipología de páginas web, se ha seleccionado la página principal de cada uno de los sitios web, la página que contiene información de los estudios que se imparten y la de contacto con la universidad.

Para el proceso de evaluación se ha utilizado la herramienta TAW. Como se ha comentado con anterioridad, TAW permite detectar errores en criterios de éxito que son analizables desde el punto de vista automático y genera una lista con las comprobaciones manuales que se deben realizar. Las herramientas de análisis automático, como TAW, ofrecen la posibilidad de detectar errores a nivel de código de programación de páginas web. Ahora bien, existen otros errores de diseño web accesible que sólo se detectan por medio de un análisis manual. Por ello, el análisis preliminar realizado con TAW se ha complementado con un análisis manual posterior.

A modo de resumen se presenta una lista de los criterios de éxito analizados. Para una mayor descripción del significado de los errores se debe consultar la WCAG 2.0 (Caldwell y otros, 2008). La Tabla 5 muestra dicha lista de criterios de éxito, con el nivel al que se refiere y el principio de diseño. 
RED. Revista de Educación a Distancia. Núm. 59, Artíc. 06, 30-10-2019

DOI: http://dx.doi.org/10.6018/red/60/06

Tabla 5 .

Descripción de los criterios de éxito analizados

\begin{tabular}{clll}
$\begin{array}{c}\text { Punto de } \\
\text { verificación }\end{array}$ & Descripción & Nivel & Principio \\
\hline 1.1 .1 & Contenido no textual & A & Perceptible \\
\hline 1.3 .1 & Información y relaciones & A & Perceptible \\
\hline 1.4 .4 & Redimensionamiento del texto & AA & Perceptible \\
\hline 2.1 .1 & Acceso completo por teclado & A & Operable \\
\hline 2.1 .3 & Acceso parcial por teclado & AAA & Operable \\
\hline 2.2 .1 & Tiempo ajustable & A & Operable \\
\hline 2.2 .4 & Interrupciones temporales & AAA & Operable \\
\hline 2.4 .10 & Encabezados de sección & AAA & Operable \\
\hline 2.4 .4 & Propósito de los enlaces (en contexto) & A & Operable \\
\hline 2.4 .7 & Foco visible & AA & Operable \\
\hline 2.4 .9 & Propósito de los enlaces (en el mismo enlace) & AAA & Operable \\
\hline 3.1 .1 & Idioma de la página & A & Comprensible \\
\hline 3.1 .4 & Abreviaturas & AAA & Comprensible \\
\hline 3.2 .2 & Introducción de datos predecible & A & Comprensible \\
\hline 3.2 .5 & Cambios bajo petición predecibles & AAA & Comprensible \\
\hline 3.3 .2 & Etiquetas en introducción de datos & A & Comprensible \\
\hline 4.1 .1 & Sintaxis de etiquetas de apertura y cierre & A & Robusto \\
\hline 4.1 .2 & Determinación del nombre de elementos de interfaz & A & Robusto \\
\hline
\end{tabular}

Nota: Elaboración propia a partir de Caldwell (2008)

El análisis se realizó de la siguiente manera:

- Fecha del estudio: mayo de 2018

- Muestra: Páginas web seleccionadas de los sitios web de las universidades.

- Metodología: Se utilizó la herramienta TAW de análisis de accesibilidad web. Se documentaron las deficiencias detectadas de manera automática y se procedió a una revisión manual de las recomendaciones de la misma herramienta. Con los resultados se estableció una clasificación numérica para poder realizar una comparación entre los diferentes sitios web.

\subsection{Resultados y niveles de accesibilidad en universidades españolas}

Los resultados obtenidos se presentan en la Tabla 6. En dicha tabla se presenta el total de errores cometidos en los diferentes principios de diseño, para cada una de las universidades analizadas. Se puede ver que los errores de Perceptibilidad y Robustez aparecen en la práctica totalidad de sitios web analizados (con las excepciones de la Universidad de les Illes Balears y de la Universidad Miguel Hernández). Todos los sitios web contienen errores de Operabilidad. El principio de diseño de Comprensibilidad ofrece los mejores resultados, con un total de cinco sitios web sin errores en el principio de diseño. En el total de todos los principios de diseño se ha detectado un total de 3710 ocurrencias de error.

Tabla 6.

Resultados del análisis

\begin{tabular}{lcccc} 
Universidad & Perceptibilidad & Operabilidad & Comprensibilidad & Robustez \\
\hline Pompeu Fabra & 118 & 307 & 17 & 43 \\
\hline Carlos III & 14 & 5 & 3 & 12 \\
\hline Politècnica de Catalunya & 5 & 119 & 1 & 129 \\
\hline
\end{tabular}

El diseño web y material didáctico accesible en la enseñanza universitaria. J. Jaume Mayol, F.J. Perales López, F. Negre Bennasar, G. Fontanet Nadal. Página 11 de 19 
RED. Revista de Educación a Distancia. Núm. 59, Artíc. 06, 30-10-2019

DOI: http://dx.doi.org/10.6018/red/60/06

\begin{tabular}{lcccc}
\hline Politècnica de València & 44 & 28 & 1 & 13 \\
\hline Autónoma de Madrid & 5 & 193 & 0 & 39 \\
\hline Cantabria & 42 & 116 & 2 & 165 \\
\hline Autónoma de Barcelona & 13 & 44 & 0 & 19 \\
\hline Rovira i Virgili & 27 & 148 & 0 & 15 \\
\hline Navarra & 101 & 137 & 18 & 40 \\
\hline Miguel Hernández de Elche & 4 & 95 & 0 & 0 \\
\hline Politécnica de Madrid & 8 & 38 & 6 & 14 \\
\hline Santiago de Compostela & 76 & 80 & 128 & 32 \\
\hline Barcelona & 14 & 19 & 3 & 211 \\
\hline València & 560 & 101 & 51 & 23 \\
\hline U. Alcalá de Henares & 59 & 154 & 0 & 31 \\
\hline U. Illes Balears & 0 & 15 & 3 & \\
\hline
\end{tabular}

Nota: Elaboración propia a partir de los resultados obtenidos

Con los indicadores de aparición de errores, y atendiendo al nivel de accesibilidad asociado a cada error, se pueden establecer los niveles de accesibilidad de cada página. En el total de las 48 páginas web analizadas, un total de 3 páginas web $(6,25 \%$ del total de páginas analizadas) alcanzan el nivel de accesibilidad AA, mientras que el resto de páginas no alcanzan ningún nivel de accesibilidad. Dichas páginas pertenecen a los sitios web de las Universidades Miguel Hernández (2 páginas) y Santiago de Compostela (1 página). En cuanto a la accesibilidad general del sitio web de la universidad, ninguno de los 16 sitios analizados alcanza el nivel mínimo de accesibilidad A.

Tabla 7.

Total de errores por sitio web y nivel de accesibilidad

\begin{tabular}{lccc} 
Abreviatura & A & AA & AAA \\
\hline UPF & 303 & 0 & 182 \\
\hline UC3M & 29 & 0 & 5 \\
\hline UPC & 141 & 0 & 113 \\
\hline UPV & 73 & 2 & 11 \\
\hline UAM & 46 & 0 & 191 \\
\hline UC & 250 & 0 & 75 \\
\hline UAB & 40 & 0 & 36 \\
\hline URV & 62 & 0 & 128 \\
\hline UNAV & 212 & 0 & 84 \\
\hline UMH & 4 & 0 & 95 \\
\hline UPM & 55 & 0 & 11 \\
\hline USC & 78 & 0 & 208 \\
\hline UB & 57 & 0 & 11 \\
\hline UV & 861 & 0 & 62 \\
\hline UAH & 82 & 0 & 154 \\
\hline UIB & 36 & 0 & 13 \\
\hline & & &
\end{tabular}

Nota: Elaboración propia a partir de los resultados obtenidos

En la Tabla 7 se muestra el total de errores de accesibilidad por sitio web y nivel de accesibilidad. El sitio web con menor número de errores en criterios de éxito de nivel A corresponde a la Universidad Miguel Hernández.

A efectos de poder comparar los resultados de los diferentes sitios web se ha establecido un procedimiento de cálculo que otorga una puntuación a partir de las ocurrencias de error en los diferentes criterios de éxito. Esta práctica es habitual en diversos sistema de evaluación automática y manual (AENOR, 2012; Benavídez, 2012; Casasola y otros,

El diseño web y material didáctico accesible en la enseñanza universitaria. J. Jaume Mayol, F.J. Perales López, F. Negre Bennasar, G. Fontanet Nadal. 
2017; Muñoz y otros, 2012; SGAD, 2018). El procedimiento de cálculo establecido se determina a partir de:

- Se calcula el total de criterios de éxito que no poseen error, por nivel de accesibilidad (A, AA, AAA). Dicha puntuación se denomina $\mathrm{PN}_{\mathrm{i}}$ (puntuación del Nivel i, donde i corresponde a los niveles A, AA o AAA) y se calcula como se especifica a continuación:

$$
\begin{aligned}
& P N_{A}=\text { total de criterios de éxito del Nivel A que no poseen error; } \\
& \text { si }\left(P N_{A}==\text { total de criterios de éxito de Nivel A analizados }\right) \text { entonces } \\
& P N_{A A}=\text { total de criterios de éxito de Nivel AA que no poseen } \\
& \text { error }
\end{aligned}
$$

Si no

$$
P N_{A A}=0
$$

Si (PNAA== número de criterios de éxito de Nivel AA analizados) entonces

$$
\begin{aligned}
& P N_{A A A}=\text { total de criterios de éxito de Nivel AAA que no poseen } \\
& \text { error }
\end{aligned}
$$

Si no

$$
P N_{A A A}=0
$$

- Para cada nivel de accesibilidad se establece la siguiente puntuación:

$$
\mathrm{VN}_{\mathrm{i}}=\mathrm{PN}_{\mathrm{i}} / \mathrm{NN}_{\mathrm{i}}
$$

Siendo:

$$
\begin{aligned}
& \mathrm{VN}_{\mathrm{i}} \text { : Valoración del Nivel i } \\
& \mathrm{PN}_{\mathrm{i}} \text { : Puntuación del Nivel i } \\
& \mathrm{NN}_{\mathrm{i}} \text { : Número de criterios de éxito de nivel i analizados }
\end{aligned}
$$

- Se establece una puntuación final de cada página, en la que intervienen las puntuaciones de cada uno de los niveles $\left(\mathrm{VN}_{\mathrm{i}}\right)$, considerando una ponderación asociada a cada nivel. De esta manera, se asigna una ponderación de 0,6 a la valoración del nivel A $\left(\mathrm{VN}_{\mathrm{A}}\right), 0,3$ al $\mathrm{VN}_{\mathrm{AA}}$ y 0,1 al $\mathrm{VN}_{\mathrm{AAA}}$. Dicha ponderación se ha establecido a partir de la importancia que poseen los controles en cada nivel de accesibilidad, pudiendo ser modificable en diversos escenarios de cálculo. De esta manera:

$$
\mathrm{PP}_{\mathrm{j}}=0,6 * \mathrm{VN}_{\mathrm{A}}+0,3 * \mathrm{VN}_{\mathrm{AA}}+0,1 * \mathrm{VN}_{\mathrm{AAA}}
$$

Siendo

$$
\begin{aligned}
& \mathrm{PP}_{\mathrm{j}} \text { : Puntuación de la página } \mathrm{PP}_{\mathrm{j}} \\
& \mathrm{VN}_{\mathrm{i}} \text { : Puntuación del Nivel } \mathrm{i}
\end{aligned}
$$


- Finalmente se establece una puntuación final del sitio web como se describe a continuación:

$\mathrm{PSW}_{\mathrm{k}}=$ Suma de $\mathrm{PP}_{\mathrm{j}}$ de páginas del sitio $/ \mathrm{NP}_{\mathrm{k}}$

Siendo

$\mathrm{PSW}_{\mathrm{k}}$ : Puntuación del sitio web

$\mathrm{PP}_{\mathrm{j}}$ : Puntuación de la página web

$\mathrm{NP}_{\mathrm{k}}$ : Número de páginas analizadas en el sitio web

Cabe destacar que el anterior procedimiento de cálculo permite establecer una clasificación a efectos de seguimiento de las normas de diseño web. Ahora bien, la obtención de una puntuación por medio del sistema de calificación anterior no asegura, en ningún caso, que una página web sea accesible: sólo propone un método para evaluar el nivel de accesibilidad de una página web.

Se ha aplicado el procedimiento a los sitios web analizados, con los resultados que se muestran en la Tabla 8. En dicha tabla se puede apreciar que los mejores resultados en cuanto a análisis de criterios de éxito corresponde a las Universidades Miguel Hernández, Santiago de Compostela y Alcalá de Henares.

Tabla 8 .

Puntuación de los sitios web

\begin{tabular}{lr} 
Universidad & Puntuación TAW 2 \\
\hline Miguel Hernández de Elche & 8,47 \\
\hline Santiago de Compostela & 6,72 \\
\hline U. Alcalá de Henares & 5,27 \\
\hline Autónoma de Madrid & 5,21 \\
\hline U. Illes Balears & 5,18 \\
\hline Autónoma de Barcelona & 5,01 \\
\hline Politècnica de València & 5,01 \\
\hline Politècnica de Catalunya & 4,72 \\
\hline Rovira i Virgili & 4,67 \\
\hline Politécnica de Madrid & 4,32 \\
\hline Barcelona & 3,92 \\
\hline Carlos III & 3,89 \\
\hline Cantabria & 3,76 \\
\hline València & 3,58 \\
\hline Navarra & 2,90 \\
\hline Pompeu Fabra & 2,30 \\
\hline
\end{tabular}

Nota: Elaboración propia a partir de los resultados obtenidos

\section{CONCLUSIONES}

A partir de los resultados y análisis anteriores, además de los antecedentes presentados, se pueden obtener algunas conclusiones:

- Existe una relación directa entre el nivel de estudios y el nivel de ocupación laboral de las personas con discapacidad, de manera que un incremento del nivel de estudios incrementa las posibilidades de inserción laboral. Dado que la población posee menores niveles de formación, entonces se puede concluir que los menores niveles de ocupación pueden venir derivados de los menores niveles

El diseño web y material didáctico accesible en la enseñanza universitaria. J. Jaume Mayol, F.J. Perales López, F. Negre Bennasar, G. Fontanet Nadal. $\quad$ Página 14 de 19 
de formación. Por tanto, una mejora de los niveles de formación podría incrementar la inserción laboral de personas con discapacidad.

- Aunque en España se han desarrollado diversas iniciativas legislativas para impulsar, desarrollar y aplicar estándares de accesibilidad en las páginas web de centros públicos universitarios, los niveles de accesibilidad de los contenidos digitales, en general, no están a los niveles que exige la legislación española (Casasola y otros, 2017).

- Los niveles de accesibilidad de los recursos electrónicos en los ámbitos universitarios analizados no facilitan que los estudiantes puedan acceder a los mismos a través de plataformas de formación online (e-learning). De esta manera, los estudiantes que poseen algún tipo de discapacidad se encuentran con obstáculos para realizar estudios universitarios, cuando, como comentan Pastor y Antón (2009), el uso de las TIC es, no sólo una necesidad, también un derecho de las personas con discapacidad y constituye un recurso imprescindible para garantizar la igualdad de oportunidades y mejorar su calidad de vida (Antón, 2010).

- Se dedica poco tiempo a la creación de entornos y espacios virtuales de educación universitarios accesibles, de manera que queda comprometida la formación en línea para personas con discapacidad. Como explican algunos autores, para evitar la discriminación, se deben tomar, entre otras medidas, el ingreso y la permanencia a los espacios virtuales (Pastor y Antón, 2009) y asegurar el acceso y uso a los recursos electrónicos.

- Se ha podido establecer un procedimiento de puntuación de sitios web a partir de la evaluación de los criterios de éxito, teniendo en cuenta el nivel de accesibilidad de cada uno de los criterios de éxito analizados. Dicho sistema de puntuación es una alternativa a otros procedimientos de cálculo.

Por tanto, parece necesario:

- Diseñar sistemas y favorecer estructuras y organizaciones accesibles, además de desarrollar mecanismos de compensación que hagan posible la educación de las personas con discapacidad, además de su participación en una comunidad inclusiva. En este sentido, la Administración y el profesorado deben adoptar un papel determinante en la realización de estudios sobre la aplicación de las directrices de accesibilidad dado que, a pesar de los avances legislativos, existen todavía muchos aspectos por mejorar (Ribera y otros, 2009; Casasola y otros 2017). Cabe señalar, no obstante, la relevancia de posibilitar formación en materia de accesibilidad al profesorado. Slater, Pearson y Forbes (2015) destacan la importancia de introducir especialistas en accesibilidad en las Facultades con el objetivo de crear conciencia y generar un cambio institucional de manera que todos los estudiantes puedan ver cubiertas sus necesidades en el diseño y producción de los contenidos.

- Mejorar las medidas de impulso y promoción de campañas de sensibilización para mejorar la accesibilidad digital. Estas medidas deberían incluir, entre otras, la formación integral que permita a los profesionales de la educación dar respuesta a las necesidades que plantean los estudiantes ya que, como explica MuñozCantero y Losada-Puente (2018), en los discursos de los docentes se dilucida falta de preparación, formación e información. Esto cobra especial importancia si se 
considera que esta situación "les está conduciendo a actitudes de desconcierto, desmotivación e incluso, pasividad" (Muñoz-Cantero y Losada-Puente, 2018, p. $54)$.

- Impulsar acciones de mejora y de concienciación para los responsables de las plataformas de formación online, y para el profesorado responsable de la generación de documentación académica, de la importancia de tener en cuenta el acceso de las personas con discapacidad. En este sentido, Kelly, Lewthawaite y Sloan (2010) defienden la necesidad de crear un marco para los responsables de las políticas educativas con el fin de evitar las dificultades que se han experimentado en el mundo desarrollado con el objetivo de influir en la creación de un enfoque más práctico y pragmático en el mundo en desarrollo.

Presentación del artículo: 7 de abril de 2019

Fecha de aprobación: 16 de octubre de 2019

Fecha de publicación: 30 de octubre de 2019

Jaume Mayol J., Perales López, F.J., Negre Bennasar, F., y Fontanet Nadal, G. (2019). El diseño web y material didáctico accesible en la enseñanza universitaria. RED. Revista de Educación a Distancia, 60. DOI: http://dx.doi.org/10.6018/red/60/06

\section{Financiación}

Esta investigación no ha recibido ninguna subvención específica de los organismos de financiación en los sectores públicos, comerciales o sin fines de lucro.

\section{REFERENCIAS}

AENOR; (2012); Norma española UNE 139803. Requisitos de accesibilidad para contenidos en la Web; Edita: Asociación Española de Normalización y Certificación; DL: M-24678:2012; Madrid, 2012

Amado-Salvatierra, H. R., González, J. H., Tortosa, S. O. (2018). Formalización de un marco metodológico para la implementación de un proyecto educativo virtual accesible. Educación XX1, 21(2).

Antón, P. (2010). Programas y apoyos técnicos para favorecer la accesibilidad en la universidad. Revista Apertura, (Especial), 6-17.

Benavídez, C; (2012); Libro blanco de eXaminator; Documentación técnica del procedimiento de cálculo de la herramienta de evaluación web Examinator, Argentina 2012

Burgstahler, S. 2002. Universal design of distance learning. Information Technology and $\begin{array}{lllll}\text { Disabilities } & 8 & (1) . & \text { Available online at }\end{array}$ www.rit.edu/ easi/itd/itdv08n1/burgstahler.htm

Caballero-Cortés, L.; Faba-Pérez, C.; Moya-Anegón, F. (2008). Evaluación comparativa de la accesibilidad de los espacios web de las bibliotecas universitarias españolas y norteamericanas. Investigación Bibliotecológica. 23(47), 45-66.

El diseño web y material didáctico accesible en la enseñanza universitaria. J. Jaume

Mayol, F.J. Perales López, F. Negre Bennasar, G. Fontanet Nadal. Página 16 de 19 
Caldwell, B; Cooper, M; Guarino, L; Vanderheiden; G; (2008); Web Content Accessibility Guidelines (WCAG) 2.0. W3C Recommendation; Publicado por World Wide Web Consortium (W3C); www.w3.org/WAI/intro/wcag

Casasola Balsells, L. A.; Guerra González, J. C.; Casasola Balsells, M. A.; Pérez Chamorro, V. A. (2017). La accesibilidad de los portales web de las universidades públicas andaluzas. Revista Española de Documentación Científica, 40(2)

Chacón-Medina, A.; Chacón-López, H.; López-Justicia, M. D.; Fernández-Jiménez, C. (2013). Dificultades en la Accesibilidad Web de las Universidades Españolas de acuerdo a la Norma WCAG 2.0. Revista Española de Documentación Científica, $36(4), 1-13$.

Chicaiza, J; Piedra, N; Valencia, M.P; (2014); Consideraciones de accesibilidad en la producción y distribución de recursos educativos en formato PDF: Un caso de implementación para la formación Virtual Accesible en América Latina; V Congreso Internacional sobre Calidad y Accesibilidad de la Formación Virtual (CAFVIR 2014); Guatemala, mayo de 2014

Eckes, Suzanne E.; Ochoa, Theresa A; (2005); Students with disabilities: Transitioning from high school to higher education. American Secondary Education, 2005, 620.

Edmonds, C. D. (2004). Providing access to students with disabilities in online distance education: Legal and technical concerns for higher education. American Journal of Distance Education, 18(1), 51-62.

Fontanet Nadal, G.; Jaume Mayol, J; (2011); Importancia y situación actual de la accesibilidad web para el turismo accesible; Revista: Pasos. Revista de turismo y patrimonio cultural; Instituto Universitario de Ciencias Políticas y Sociales; Volumen: 9 Número: 2; España; ISSN: 1695-7121.

Fraiz, J.A; Alén, E; Domínguez, T; (2008); Un nuevo desafío para la Web: el contenido sobre accesibilidad en las Web turísticas oficiales de las Comunidades Autónomas. VII Congreso Nacional de Turismo y Tecnologías de la Información y las Comunicaciones - TURITEC 2008, Málaga, octubre de 2006. Ed: Universidad de Turismo (Universidad de Málaga).

Guash, D; (2010); La accesibilidad del entorno universitario y su percepción por parte de los estudiantes con discapacidad, observatorio universal y discapacidad; Edita: Observatorio Universidad y Discapacidad, Fundación ONCE y Universidad Politécnica de Cataluña - Barcelona Tech; ISBN: 978-84-7653-502-8

Hilera, J.R.; Fernández, L.; Suárez, E.; Vilar, E.T. (2013). Evaluación de la accesibilidad de páginas web de universidades españolas y extranjeras incluidas en rankings universitarios internacionales. Revista Española de Documentación Científica, 36 (1), 1-16.

Hilera, J.R; Campo, E; (2015); Guía para crear contenidos digitales accesibles. Documentos, presentaciones, vídeos, audios y páginas web; Servicio de Publicaciones de la Universidad de Alcalá, ISBN: 978-84-16133-52-9; DL: M2798-2015

Jaume, J.; Fontanet Nadal, G.; Bibiloni Coll, A; (2011); Análisis y procedimiento de mejora de la accesibilidad web; Revista Ibérica de Sistemas y Tecnologías de Información, ISSN: 1646-9895, Número: 7, 06/2011.

El diseño web y material didáctico accesible en la enseñanza universitaria. J. Jaume

Mayol, F.J. Perales López, F. Negre Bennasar, G. Fontanet Nadal. Página 17 de 19 
Jiménez, J; Chao, J; Bibiloni, T; Fontanet, G; (2010); Dynamic Generation and Visualization of Customized Touristic Packages; The Fisrt International Conferences on Tourism between China-Spain, March 28-31, 2010, Palma Spain. Editorial Pearson Digital.

Kelly, B., Lewthwaite, S., Sloan, D. (2010). Developing countries; developing experiences: approaches to accessibility for the real world. In Proceedings of the 2010 international cross disciplinary conference on web accessibility (W4A) (p. 3). ACM.

Lance, G. D. 2002. Distance learning and disability: A view from the in-structor's side of the virtual lectern. Information Technology and Disabilities.

Muntaner, J. J., Perales, F. J., Negre, F., Varona, J., Manresa-Yee, C. (2008). Sistema de interacción natural avanzado (SINA): Proceso de mejora y ajuste para usuarios con parálisis cerebral y esclerosis múltiple. En M. D. Hurtado F.J. Soto, La igualdad de oportunidades en el mundo digital, (139-151). Murcia, España: Consejería de Educación, Ciencia e Investigación

Muñoz, E y otros; (2012); Metodología del Observatorio de Accesibilidad Web UNE 139803:2012; Observatorio de Accesibilidad del Ministerio de Hacienda y Administraciones Públicas

Muñoz-Cantero, J. M., Losada-Puente, L. (2018). Implicaciones de la actitud docente en la calidad de vida del alumnado con alteraciones del desarrollo intelectual. Educación XX1, 21(2), 37-58.

López, J; (2010); Las principales barreras de accesibilidad web en la educación para personas con discapacidad; Revista ISEES (Inclusión Social y Equidad en la Educación Superior), número 7, Julio 2010, páginas 133-142; Santiago de Chile

Luque, D. J., Rodríguez, G. Romero, J. F. (2005). Accesibilidad y Universidad. Un estudio descriptivo Accessibility and University. A descriptive study. Intervención Psicosocial, 14(2), 209-222.

ODISMET, Observatorio sobre discapacidad y mercado de trabajo en España; (2016); Observatorio sobre Discapacidad y Mercado de Trabajo de la Fundación ONCE; Fundación ONCE

Pastor, C. A, Antón Ares, P. (2008), Aprendizaje permanente del profesorado y TIC. Una experiencia de cooperación al desarrollo en Nicaragua, Paraguay y República Dominicana, Revista Latinoamericana de Tecnología. Educativa, vol. 7, núm. 1, pp. 97-106.

Ribera, M., Térmens, M., Frías, A. (2009). La accessibilidad de las webs de las universidades españolas. Balance 2001-2006. Revista Española de Documentación Científica, 32(3), 66-88.

Saldarriaga, J.A; (2014); Accesibilidad web, una estrategia para la inclusión educativa en entornos virtuales de eduación; Primer Congreso Internacional Virtual en Discapacidad y Derechos Humanos, Argentina, diciembre de 2014

Sam-Anlas, C. A. y Stable-Rodríguez, Y. (2016). Evaluación de la accesibilidad web de los portales del Estado en Perú. Revista Española de Documentación Científica, 39

El diseño web y material didáctico accesible en la enseñanza universitaria. J. Jaume

Mayol, F.J. Perales López, F. Negre Bennasar, G. Fontanet Nadal. Página 18 de 19 
Sama, V; Sevillano, E; (2012); Universidad sin barreras. Guía de accesibilidad de documentos electrónicos; Universidad Nacional de Educación a Distancia; ISBN: 978-84-362-5669-7; DL: M-21092-2012; Madrid 2012.

Serrano, E; (2009); Herramientas para la evaluación de la accesibilidad Web. Documentación de las Ciencias de la Información 2009, 32: 245-266, Universidad de Alcalá.

SGAD - Secretaría General de Administración Digital; (2018); Metodología del Observatorio de Accesibilidad Web UNE 139803:2012, versión 2; Ministerio de Hacienda y Función Pública; NIPO: 169-18-061-1

SGAD2 - Secretaría General de Administración Digital; (2018); Guía de adaptación WCAG 2.1 desde WCAG 2.0; Ministerio de Hacienda y Función Pública; NIPO MPTFP: 277-19-045-3; NIPO MINHAC: 185-19-102-7

Slater, R., Pearson, V. K., Warren, J. P., Forbes, T. (2015). Institutional change for improving accessibility in the design and delivery of distance learning-the role of faculty accessibility specialists at The Open University. Open Learning: The Journal of Open, Distance and e-Learning, 30(1), 6-20.

Térmens, M; Barrios, M; Días M; Guasch, D; Ponsa, P; Ribera, M; (2008); Estudio de la accesibilidad de los documentos científicos en soporte digital; Revista Española de Documentación Científica 31, 4, Octubre-Diciembre, 552-572; ISSN: 02100614; doi: 10.3989/redc.2008.4.651

Toledo, P., Sánchez, J. M., Gutiérrez, J. J. (2013). Evolución de la accesibilidad web en las universidades andaluzas. Píxel-Bit. Revista de Medios y Educación, (43), 6583.

Tuero, E., Cervero, A., Esteban, M., Bernardo, A. (2018). ¿Por qué abandonan los alumnos universitarios? Variables de influencia en el planteamiento y consolidación del abandono. Educación XX1, 21(2).

Zubillaga del Río, Ainara (2010). La Accesibilidad como elemento del proceso educativo: Análisis del Modelo de Accesibilidad de la Universidad Complutende de Madrid para atender las necesidades de los estudiantes con discapacidad. Tesis doctoral. Universidad Complutense de Madrid, España. (T32369) 